\title{
Preparation and Magnetic Comparison of Silane-Functionalized Magnetite Nanoparticles
}

\author{
He Quanguo*, Zeng Lei, Wu Wei, Hu Rong and Huang Jingke \\ Green Packaging and Biological Nanotechnology Laboratory, \\ Hunan University of Technology, Zhuzhou 412008, China
}

(Received May 22, 2009; accepted November 18, 2009)

Key words: magnetic nanocomposites, magnetic properties, silance modification, magnetite nanoparticles, surface functionalization

Silane-modified magnetite nanoparticles (NPs) were synthesized by chemical coprecipitation and subsequent surface modification with 3-aminopropyltriethyloxysilane (APTES) and mercaptopropyltriethoxysilane (MPTES). Functionalized $\mathrm{Fe}_{3} \mathrm{O}_{4}$ nanospheres with an average diameter of $25 \pm 5 \mathrm{~nm}$ were clearly characterized by good dispersion, good morphology, accurate composition, and excellent optical properties, as well as maximized saturation magnetization. The results confirm that the functionalized NPs retain most of their original saturation magnetization. The saturation magnetization $\left(M_{\mathrm{s}}\right)$ of the $\mathrm{Fe}_{3} \mathrm{O}_{4}$ NPs was found to be $64.4 \mathrm{emu} \mathrm{g} \mathrm{g}^{-1}$ at $300 \mathrm{~K}$ and $71.8 \mathrm{emu} \mathrm{g}^{-1}$ at $5 \mathrm{~K}$. Likewise, the APTES-coated and MPTES-coated $\mathrm{Fe}_{3} \mathrm{O}_{4}$ NPs demonstrated slight decreases in their saturation magnetization and paramagnetism at $300 \mathrm{~K}$, and the decrease in magnetic property agreed with previous reports. Therefore, the negligible loss in magnetic property indicates the potential of these silane-functionalized magnetite NPs for further biochemical manipulation and assembly on surface-tailored NPs for biosensor applications.

\section{Introduction}

Surface-layered magnetic iron oxide nanoparticles (NPs) have been the focus of many investigations in recent years owing to their interesting size- and shape-dependent properties. By passivating their surface properly, these NPs become analogous to single magnetic nanocrystals, thereby extending their application owing to their interior magnetic properties, since their surface layers can bring about other different properties including stability in colloidal solution, improved biocompatibility and hydrophilicity, and low cytotoxicity. Therefore, the surface passivation of NPs using organic molecules or nonmetals such as silica and silane ${ }^{(1)}$ has recently gained increasing attention among many research groups. Surface-layered magnetic iron oxide NPs are useful for several potential applications such as the design of multifunctional catalysts and biosensors, magnetic resonance imaging (MRI), target drug delivery, and bioseparation. ${ }^{(2-5)}$

"Corresponding author: e-mail: hequanguo@126.com 
Particularly, magnetite $\left(\mathrm{Fe}_{3} \mathrm{O}_{4}\right)$ NPs have attracted much attention owing to their wide biomedical applications. ${ }^{(6,7)}$ To be properly applied in biomedicine, $\mathrm{Fe}_{3} \mathrm{O}_{4}$ NPs should exhibit biocompatibility, paramagnetism, specific targeting capability, and stability in aqueous solution. Owing to their excellent surface energy and magnetic properties, $\mathrm{Fe}_{3} \mathrm{O}_{4}$ NPs are thermodynamically unstable and prone to agglomeration, which is one of the critical obstacles in producing stable and highly monodisperse magnetic NPs. Thus, a modification technique is frequently employed using the interaction between the magnetic NP surface and functional groups of organic molecules. ${ }^{(8)}$ Also, the formation of a coating on magnetic NPs could serve as a protective layer against oxidation and some extreme chemical environments, and could be applied to further derivative conjugations towards biosensors. Consequently, tailing a magnetic NP surface is a popular challenge in the realization of these purposes.

Herein, the silane agents APTES and MPTES were considered as candidates for the direct surface modification of $\mathrm{Fe}_{3} \mathrm{O}_{4} \mathrm{NPs}$ (shown in Fig. 1), owing to the advantages of biocompatibility as well as high density of the surface functional groups $-\mathrm{NH}_{2}$ and $-\mathrm{SH}$, which allow further surface derivation of these NPs for bioapplications. In this study, APTES-coated and MPTES-coated $\mathrm{Fe}_{3} \mathrm{O}_{4}$ NPs were prepared using simple procedures. They exhibited good dispersion and good stability in aqueous solutions. After surface silane tailoring, the modified magnetite NPs still maintained the structure and magnetic properties of the bare NPs. The preparation and surface functionality modification of these magnetic NPs led to our success in versatile surface tailoring techniques, and opened up new avenues for magnetic-NP-based specific biosensing applications.

\section{Experimental}

\subsection{Materials}

Ferric chloride $\left(\mathrm{FeCl}_{3} \cdot 6 \mathrm{H}_{2} \mathrm{O}, 99.0 \mathrm{wt} \%\right)$ was purchased from Tianjin Bodi Chemicals Co., Ltd., and ferrous chloride $\left(\mathrm{FeCl}_{2} \cdot 4 \mathrm{H}_{2} \mathrm{O}, 99.0 \mathrm{wt} \%\right)$ from Tianjin Shuangchuan Chemicals Co., Ltd. APTES and MPTES were purchased from Sigma. Sodium

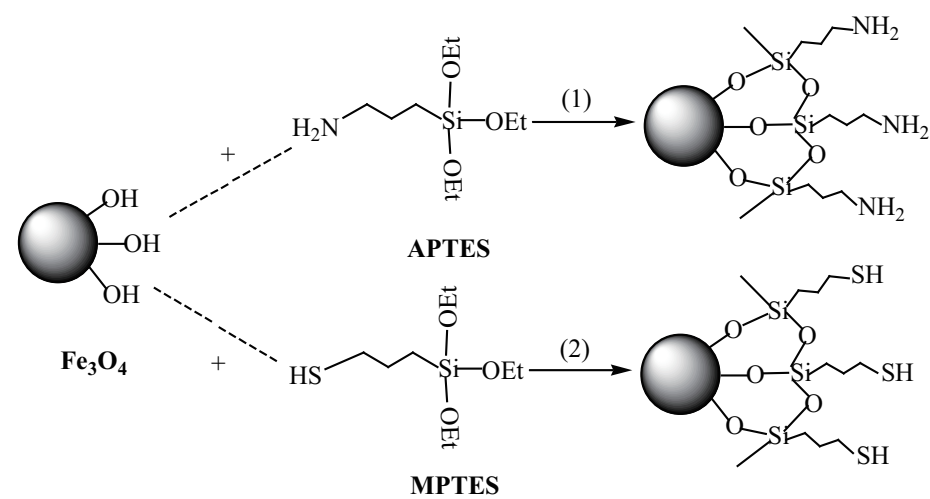

Fig. 1. Scheme of surface functionalization of magnetite NPs: (1) amino coating and (2) thiolation. 
hydroxide $(\mathrm{NaOH})$ was purchased from Hunan Huihong Chemicals Co., Ltd. All these chemicals were of analytical grade and used as received. Water (18.2 M $\Omega$ ) was purified using SUPER WATER-II water purification systems. The NdFeB magnet used was purchased from the local market; it was used for separating magnetic particles during washing and selection.

\subsection{Synthesis of surface-functionalized magnetite NPs}

Synthesis of magnetic NPs: Magnetite nanocrystals as seeds were prepared through the chemical coprecipitation of $\mathrm{Fe}$ (II) and $\mathrm{Fe}$ (III) chlorides [Fe (II)/Fe (III) ratio $=0.5]$ with $0.75 \mathrm{M} \mathrm{NaOH}$. The black precipitate was collected on a magnet, and rinsed with water several times until its $\mathrm{pH}$ reached 7.

Synthesis of APTES-coated magnetite NPs: A solution of APTES was added to the $\mathrm{Fe}_{3} \mathrm{O}_{4}$ suspension ( $5 \mathrm{mg}$ of $\mathrm{Fe}_{3} \mathrm{O}_{4} / \mathrm{ml}$ of ethanol solution), and the mixture was stirred in $\mathrm{N}_{2}$ atmosphere at $25^{\circ} \mathrm{C}$ for $4 \mathrm{~h}$. The APTES-coated $\mathrm{Fe}_{3} \mathrm{O}_{4} \mathrm{NPs}$ were collected with a magnet, and washed with $50 \mathrm{~mL}$ of ethanol and then with deionized water three times.

Synthesis of MPTES-coated magnetite NPs: Similarly to the above procedures, the preparation of the MPTES-coated $\mathrm{Fe}_{3} \mathrm{O}_{4}$ NPs involved the following processes: a solution of MPTES was added to the $\mathrm{Fe}_{3} \mathrm{O}_{4}$ suspension $\left(5 \mathrm{mg}\right.$ of $\mathrm{Fe}_{3} \mathrm{O}_{4} / \mathrm{ml}$ of ethanol solution), and the mixture was stirred in $\mathrm{N}_{2}$ atmosphere at $25^{\circ} \mathrm{C}$ for $4 \mathrm{~h}$. The MPTESmodified $\mathrm{Fe}_{3} \mathrm{O}_{4} \mathrm{NPs}$ were collected with a magnet and washed with $50 \mathrm{~mL}$ of ethanol and then with deionized water three times.

\subsection{Characterization}

Information on the size distribution, morphologies, structures, and compositions of the samples was obtained using a nanosizer, transmission electron microscopy (TEM), X-ray powder diffraction (XRD) analysis, energy dispersive spectroscopy (EDS), and X-ray photoelectron spectroscopy (XPS). The composition study was carried out on an EDS (from EMAX) of a Hitachi 3000N microscope operated at 25 $\mathrm{kV}$, where sample solutions were deposited onto an Al substrate at ambient temperature. TEM was performed on a JEM-1230 electron microscope $(20 \mathrm{kV})$. The NP samples dispersed in ethanol solution were cast onto a carbon-coated copper grid sample holder and dried by evaporation at room temperature. Size distribution was recorded on a nanosizer (ZEN3600). The structural planes of the products were identified by XRD analysis. Powder diffraction patterns were recorded on a Bruker Advanced-D8 powder diffractometer equipped with a $\mathrm{Si}(\mathrm{Li})$ solid-state detector and a $\mathrm{Cu} \mathrm{K} \alpha$ radiation source $(\lambda=1.5418 \AA)$. Data were collected from $2 \theta=5^{\circ}$ to $90^{\circ}$ at scan rates of $0.02^{\circ}$ per step and $5 \mathrm{~s}$ per point. XPS was conducted on a Kratos Axis Ultra DLD. This system used a focused monochromatic Al X-ray $(1486.6 \mathrm{eV})$ source for excitation and a spherical section analyzer. The percentages of individual elements detected were determined from the relative composition analysis of the peak areas of the bands. The relative peak areas and their corresponding sensitivity factors were used to obtain relative compositions.

The optical and magnetic properties were determined using ultraviolet visible spectra (UV-Vis) and a superconducting quantum inteference device (SQUID). UV-Vis spectras were acquired on a Purkinje general T-1901 spectrophotometer and collected in the range 
of 190-800 nm. Magnetic measurement was performed on a SQUID magnetometer (Quantum Design MPMS XL-7). Magnetic susceptibility M-H curves were obtained at $T=300$ and $5 \mathrm{~K}$, where $M$ is the magnetization and $H$ is the magnitude of the applied magnetic field, which were measured as a function of temperature in a magnetic field.

\section{Results and Discussion}

\subsection{Characterization of functionalized $\mathrm{Fe}_{3} \mathrm{O}_{4} \mathrm{NPS}$}

Generally, $\mathrm{Fe}_{3} \mathrm{O}_{4}$ is prepared via the coprecipitation of $\mathrm{Fe}^{2+}$ and $\mathrm{Fe}^{3+}$ ion mixtures under alkaline condition. The synthetic chemistry of the formation of magnetites is illustrated by the following reaction:(9)

$$
\mathrm{Fe}^{2+}+2 \mathrm{Fe}^{3+}+8 \mathrm{OH}^{-} \rightarrow \mathrm{Fe}_{3} \mathrm{O}_{4} \downarrow+4 \mathrm{H}_{2} \mathrm{O}
$$

Theoretically, the complete precipitation of $\mathrm{Fe}_{3} \mathrm{O}_{4}$ is expected in the $\mathrm{pH}$ range of 7.514 , when the molar ratio of $\mathrm{Fe}$ (II) to $\mathrm{Fe}$ (III) is $1: 2$. For practical purposes, a mixture of $\mathrm{Fe}^{2+} / \mathrm{Fe}^{3+}$ ions with a molar ratio of $1: 1$ was also used to prepare magnetite since $\mathrm{Fe}^{2+}$ is prone to oxidation in aqueous media. However, we successfully prepared magnetite NPs by modified chemical coprecipitation with the addition of excess amounts of sodium hydroxide as compared with that obtained at the theoretical ratio in eq. (1). The $\mathrm{Fe}_{3} \mathrm{O}_{4}$ NPs prepared via liquid phase reaction under alkaline condition possess many hydroxyl ions $\left(\mathrm{OH}^{-}\right)$, and the NPs dispersed in water can adsorb hydroxyl $\left(\mathrm{OH}^{-}\right)$and hydrogen $\left(\mathrm{H}^{+}\right)$ions from the water, thus, the surface of the magnetite NPs is covered with a sufficient number of hydroxyl (-OH) groups. ${ }^{(10)}$ Consequently, the silane agent (APTES or MPTES) could be immobilized covalently on the surface of $\mathrm{Fe}_{3} \mathrm{O}_{4} \mathrm{NPs}$ through silanization with hydroxyl groups, as shown in Fig. 1.

Figure 2 shows the diameters of the $\mathrm{Fe}_{3} \mathrm{O}_{4}(1)$, APTES- $\mathrm{Fe}_{3} \mathrm{O}_{4}(2)$, and MPTES$\mathrm{Fe}_{3} \mathrm{O}_{4}(3)$ NPs. Accordingly, the average diameter of the unmodified $\mathrm{Fe}_{3} \mathrm{O}_{4} \mathrm{NPs}$ was about $20 \mathrm{~nm}$ and that of the APTES-modified $\mathrm{Fe}_{3} \mathrm{O}_{4} \mathrm{NPs}$ was nearly $24 \mathrm{~nm}$, in comparison with that of the MPTES-modified $\mathrm{Fe}_{3} \mathrm{O}_{4} \mathrm{NPs}$, at approximately $30 \mathrm{~nm}$, which was slightly larger than that of the APTES-modified $\mathrm{Fe}_{3} \mathrm{O}_{4}$ NPs. These results suggest that the diameter slightly increased with silane functionalization.

Figure 3 shows representative TEM images of the bare (A), APTES-coated (B), and MPTES-coated (C) $\mathrm{Fe}_{3} \mathrm{O}_{4}$ NPs. The bare $\mathrm{Fe}_{3} \mathrm{O}_{4}$ NPs have an average size of ca. $25 \pm 5$ $\mathrm{nm}$ and appear spherical. Surface functionalization maintained the morphologies of the $\mathrm{Fe}_{3} \mathrm{O}_{4}$ NPs though crosslinking to some degree, which was observed to be due to silane interaction, and the average diameter of these NPs is clearly increased as a result of APTES or MPTES modification. By comparing Fig. 3(B) with Fig. 3(C), we observed that the MPTES-functionalized NPs were easily agglomerated, causing a mass of crosslinked NPs.

To confirm the composition of the NPs, EDS profiles were collected in situ and the results are shown in Fig. 4. The profiles in Fig. 4 clearly show different horizontal ordinates of different elements that are contained in the NP samples. In Fig. 4(A), the EDS profile validates the presence of $\mathrm{Fe}$ and $\mathrm{O}$ elements in the sample. The $\mathrm{Fe}: \mathrm{O}$ atom 


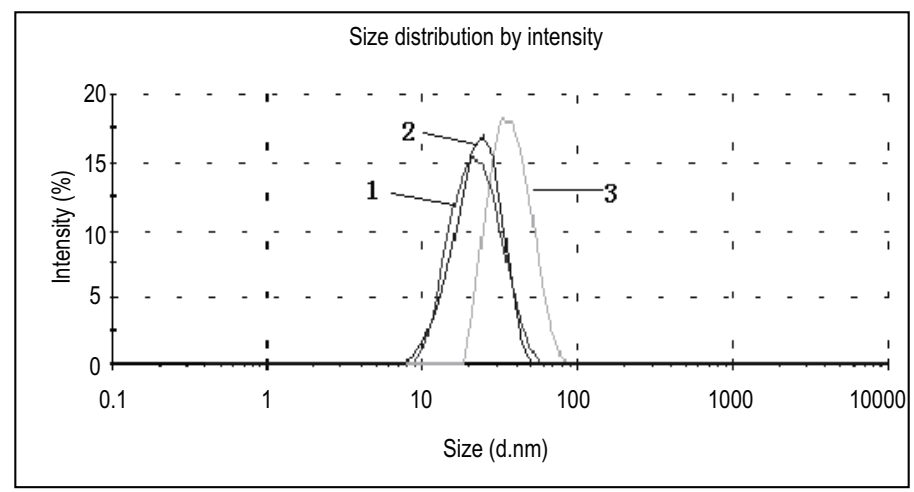

Fig. 2. Size distributions of bare (1), APTES-coated (2), and MPTES-coated (3) $\mathrm{Fe}_{3} \mathrm{O}_{4} \mathrm{NPs}_{\text {. }}$
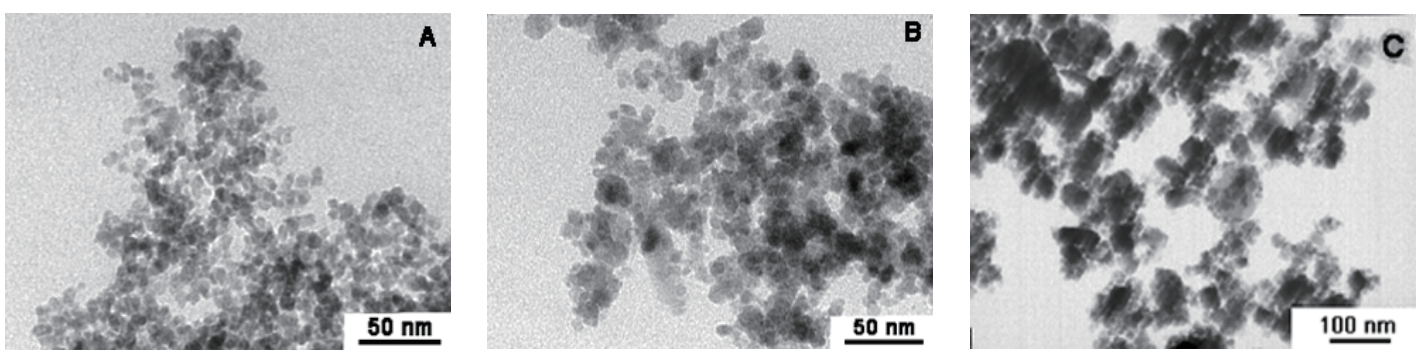

Fig. 3. TEM images of bare (A), APTES-coated (B), and MPTES-coated (C) $\mathrm{Fe}_{3} \mathrm{O}_{4} \mathrm{NPs}_{\text {. }}$

ratio is $36.37: 52.59$, which is very close to $3: 4$, confirming that the composition of iron oxide NPs is close to that of $\mathrm{Fe}_{3} \mathrm{O}_{4}$. In Fig. 4(B), the EDS profile confirms the presence of $\mathrm{Fe}, \mathrm{O}, \mathrm{Si}$, and $\mathrm{N}$ elements, and the $\mathrm{N} / \mathrm{Si}$ atom ratio is similar to $1: 1(6.59 \%: 5.75 \%)$. This result further confirms that APTES is covalently linked to the magnetite NP surface. In Fig. 4(C), the EDS profile validates the presence of $\mathrm{Fe}, \mathrm{O}, \mathrm{Si}$, and $\mathrm{S}$ elements, and the $\mathrm{S} / \mathrm{Si}$ atom ratio is close to $1: 1(1.02 \%: 1.16 \%)$; this confirms the presence of a thiolated surface structure, indicating that MPTES is covalently linked to the magnetite NP surface. Although the chemical structures are analogous for APTES and MPTES, the Si content of the APTES-functionalized NPs $(5.75 \%)$ is higher than that of the MPTESfunctionalized NPs $(1.16 \%)$, revealing that APTES is easier to anchor onto the NP surface.

The relative composition of the functionalized NP surface was further analyzed by XPS, and representative results are shown in Fig. 5. A very high carbon concentration (51.7\% atom percentage) is found on the $\mathrm{Fe}_{3} \mathrm{O}_{4}$ NP surface, as shown in Fig. 5(A), owing to the presence of carbon contamination with a binding energy $\left(E_{\mathrm{b}}\right)(284.5 \mathrm{eV})$ for $\mathrm{C}_{1 \mathrm{~s}}$ as the internal reference. In Fig. 5(B), the $E_{\mathrm{b}}$ of $\mathrm{Fe}_{2 \mathrm{p} 3 / 2}$ (curve 1) was $710.9 \mathrm{eV}$, which is close to the standard $E_{\mathrm{b}}$ data of $\mathrm{Fe}_{3} \mathrm{O}_{4}(710.5 \mathrm{eV})$. The amino-coated (curve 2) and thiolated (curve 3) $\mathrm{Fe}_{3} \mathrm{O}_{4}$ showed a lower shift in binding energy. In Fig. 5(C), the peak 

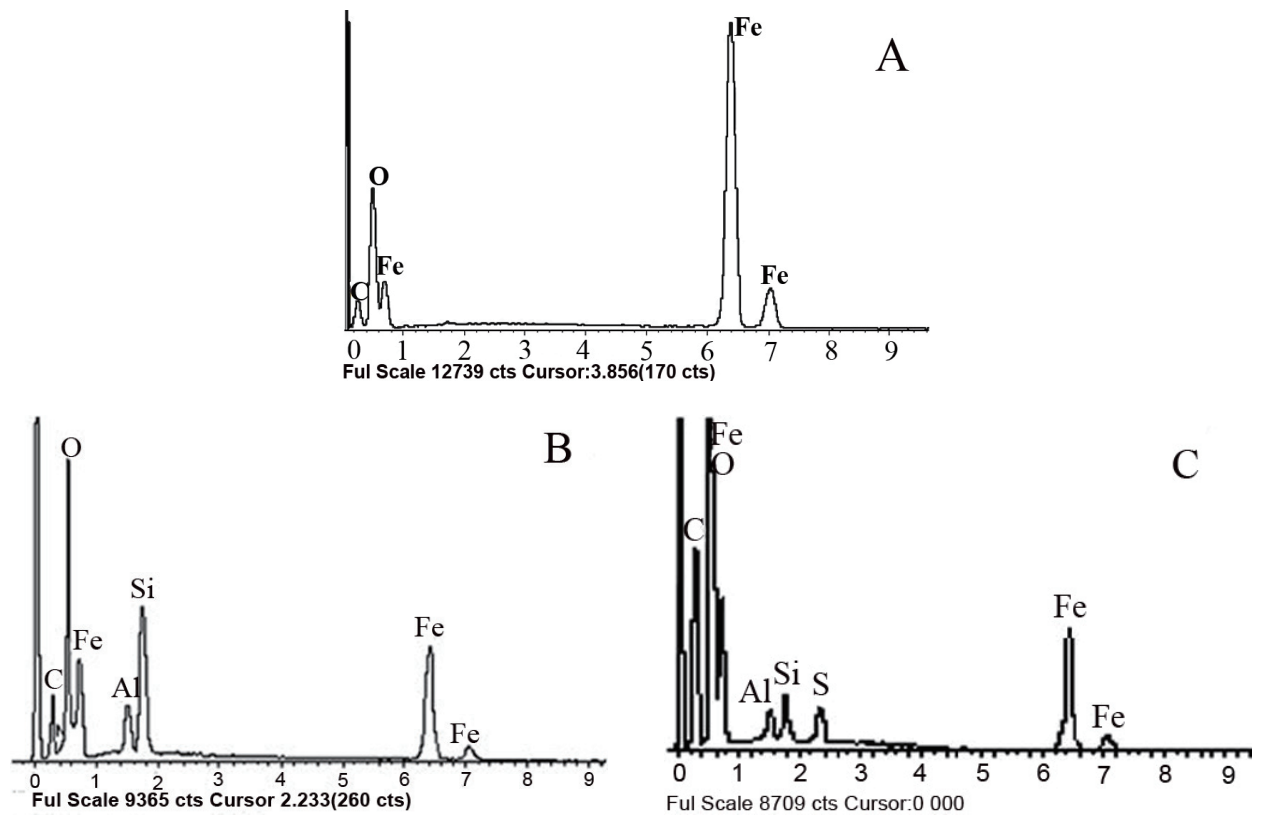

Fig. 4. Representative EDS profiles of bare (A), APTES-coated (B), and MPTES-coated (C) $\mathrm{Fe}_{3} \mathrm{O}_{4}$ NPs.

of carbon at $284.9 \mathrm{eV}$ is close to the $E_{\mathrm{b}}$ of a long-chain alkyl, which unequivocally shows that it comes from the long-chain alkyl of APTES or MPTES, and the corresponding signal intensity increased as compared with that in the case of bare $\mathrm{Fe}_{3} \mathrm{O}_{4} \cdot{ }^{(11)}$ In Fig. 5(D), the $E_{\mathrm{b}}$ of $\mathrm{Si}_{2 \mathrm{p}}$ was $102.5 \mathrm{eV}$, which is close to the standard $E_{\mathrm{b}}$ data of silane (101.9 $\mathrm{eV}$ ). In Fig. 5(E), the $E_{\mathrm{b}}$ of $\mathrm{N}_{1 \mathrm{~s}}$ was $399.5 \mathrm{eV}$ and that of $\mathrm{S}_{2 \mathrm{p}}$ was $164.1 \mathrm{eV}$, which is close to the standard $E_{\mathrm{b}}$ data of the N-C group (399.8 eV) and S-C group (163.8 eV), respectively. The above results reveal that the silane coating of the NPs is a success. ${ }^{(12)}$

In Fig. 6, the XRD spectra of bare and surface-functionalized $\mathrm{Fe}_{3} \mathrm{O}_{4}$ NPs were compared. The data (all curves) show diffraction peaks at $2 \theta=18.6^{\circ}, 30.4^{\circ}, 35.7^{\circ}, 37.3^{\circ}$, $43.2^{\circ}, 53.7^{\circ}, 57.3^{\circ}$, and $62.8^{\circ}$, which can be indexed to the (111), (220), (311), (222), (400), (422), (511), and (440) planes, respectively. Evidently, the structure did not change since all the samples' XRD spectra follow the standard $\mathrm{Fe}_{3} \mathrm{O}_{4}$ crystal patterns, and the diffraction lines are narrow to some degree, confirming that the NP size increases after surface silane modification. We used the Scherer formula (eq. (2)) to calculate particle characteristic size for comparison of the differences caused by silane coating:

$$
D=\frac{K \lambda}{\beta_{\mathrm{HKL}}^{\mathrm{s}} \cos \theta_{\mathrm{HKL}}},
$$



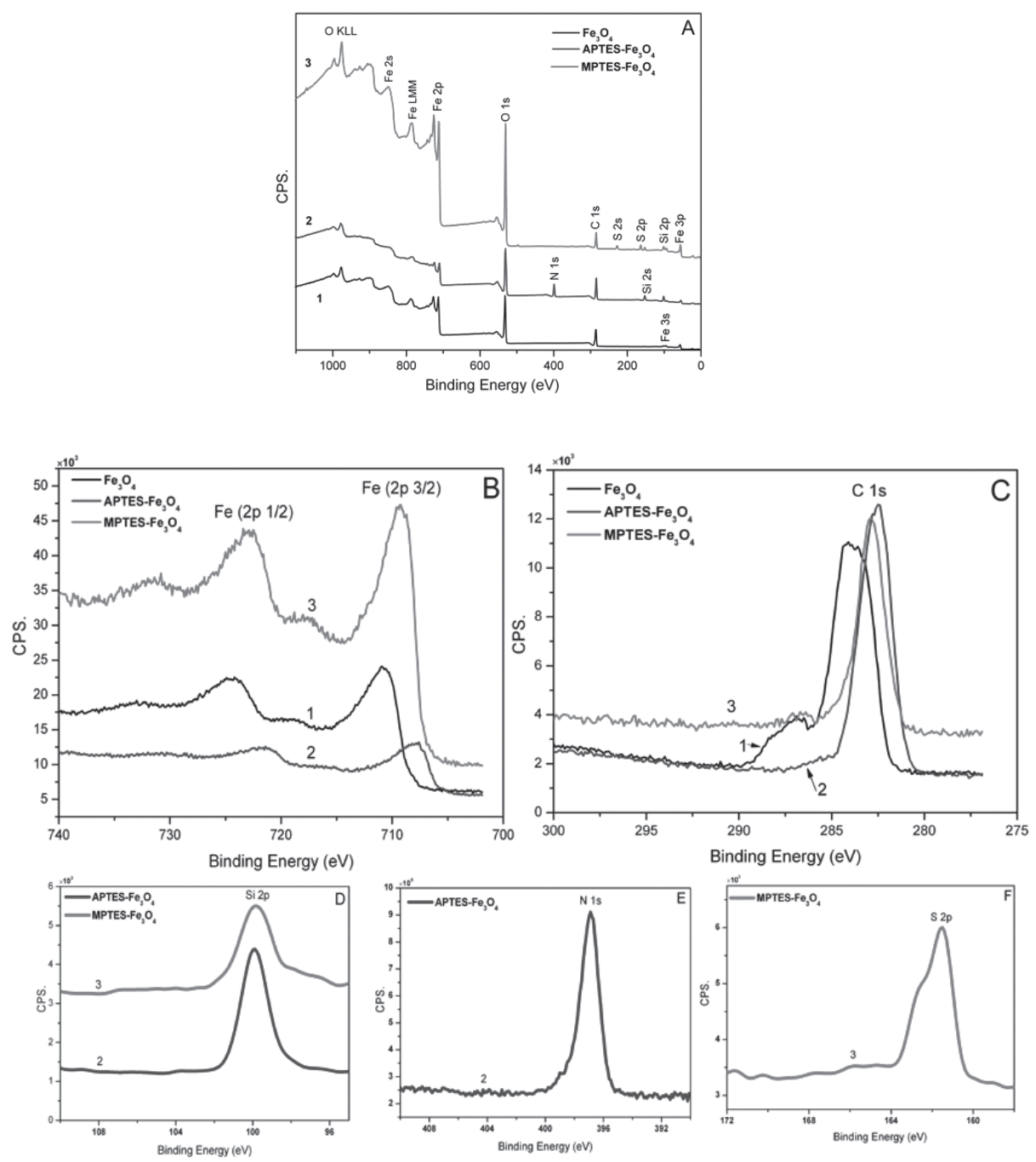

Fig. 5. (A) XPS wide-scan profiles of bare (1), APTES-coated (2), and MPTES-coated (3) $\mathrm{Fe}_{3} \mathrm{O}_{4}$ NPs, and the regions for (B) Fe(2p), (C) C(1s), (D) Si(2p), (E) N(1s), and (F) S(2p).

where $D$ is the crystal diameter, $\beta_{\mathrm{HKL}}^{\mathrm{s}}$ is the HKL diffraction line width or integral width, $\lambda$ is the wavelength of the X-radial, $\theta_{\mathrm{HKL}}$ is the angle of the diffraction peaks, and $\mathrm{k}$ is a proportion constant. The average diameter of the surface-functionalized $\mathrm{Fe}_{3} \mathrm{O}_{4} \mathrm{NPs}$ slightly increases, which might contribute to the surface functionalization of silane coating. 


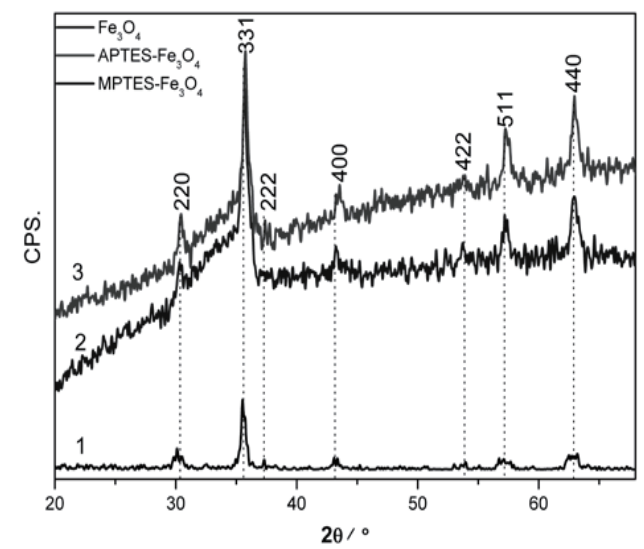

Fig. 6. XRD spectra of bare (1), APTES-coated (2), and MPTES-coated (3) $\mathrm{Fe}_{3} \mathrm{O}_{4} \mathrm{NPs}$.

\subsection{Optical properties}

The functionalized $\mathrm{Fe}_{3} \mathrm{O}_{4}$ NPs were characterized using UV-Vis absorption spectra and further compared in terms of their optical properties, as shown in Fig. 7. There is no observable surface plasmon (SP) peak of $\mathrm{Fe}_{3} \mathrm{O}_{4}$ or silane-coated $\mathrm{Fe}_{3} \mathrm{O}_{4}$, and no clear difference among the three curves for the bare $\mathrm{Fe}_{3} \mathrm{O}_{4}$ or silane-coated $\mathrm{Fe}_{3} \mathrm{O}_{4} \mathrm{NPs}$. Particularly, the absorption of magnetite NPs is highly consistent with the results of optical properties investigations of magnetite a few decades ago. ${ }^{(13,14)}$ Silane-coated $\mathrm{Fe}_{3} \mathrm{O}_{4} \mathrm{NPs}$ are distinctively different from the metal-coated $\mathrm{Fe}_{3} \mathrm{O}_{4}$ NPs. ${ }^{(15)}$ Comparison of the APTES-coated $\mathrm{Fe}_{3} \mathrm{O}_{4}$ NPs with $\mathrm{Fe}_{3} \mathrm{O}_{4}$ NPs coated with the same concentration of MPTES shows that the absorption of the former intensity is higher than that of the latter, indicating that the MPTES-coated $\mathrm{Fe}_{3} \mathrm{O}_{4}$ NPs have an excellent hypochromic effect. However, a distinct hypochromic effect is better for DNA detection; therefore, an interactive hypochromic effect resulting from surface tailoring of magnetic NPs plays an important role in their bioapplications. ${ }^{(16)}$

\subsection{Magnetic properties}

SQUID magnetometry reveals that overlaying the $\mathrm{Fe}_{3} \mathrm{O}_{4} \mathrm{NP}$ surface with a shell of APTES or MPTES causes a negligible decrease in magnetic property. Figure 8(A) shows the hysteresis loops measured at $T=300 \mathrm{~K}$ (close to room temperature) and $T$ $=5 \mathrm{~K}$ for the surface-functionalized and bare $\mathrm{Fe}_{3} \mathrm{O}_{4} \mathrm{NPs}$, respectively. The saturation magnetization $\left(M_{\mathrm{s}}\right)$ of $\mathrm{Fe}_{3} \mathrm{O}_{4} \mathrm{NPs}$ was found to be $64.4 \mathrm{emu} \mathrm{g}^{-1}$ at $300 \mathrm{~K}^{2}$ and $71.8 \mathrm{emu} \mathrm{g}^{-1}$ at $5 \mathrm{~K}$. Like the $\mathrm{Fe}_{3} \mathrm{O}_{4} \mathrm{NPs}$, the APTES-coated $\mathrm{Fe}_{3} \mathrm{O}_{4}$ NPs are almost paramagnetic at $300 \mathrm{~K}$, although its $M_{\mathrm{s}}$ was found to be $63.7 \mathrm{emu} \mathrm{g} \mathrm{g}^{-1}$ at $300 \mathrm{~K}$ and $70.3 \mathrm{emu} \mathrm{g}^{-1}$ at $5 \mathrm{~K}$. The MPTES-coated $\mathrm{Fe}_{3} \mathrm{O}_{4}$ NPs are almost paramagnetic at $300 \mathrm{~K}$, and its $M_{\mathrm{s}}$ was found to be $61.3 \mathrm{emu} \mathrm{g}^{-1}$ at $300 \mathrm{~K}$ and $71.8 \mathrm{emu} \mathrm{g}^{-1}$ at $5 \mathrm{~K}$. Evidently, the $M_{\mathrm{s}}$ at $300 \mathrm{~K}$ is lower than that at $5 \mathrm{~K}$, which agrees with the concept that increasing the temperature would 


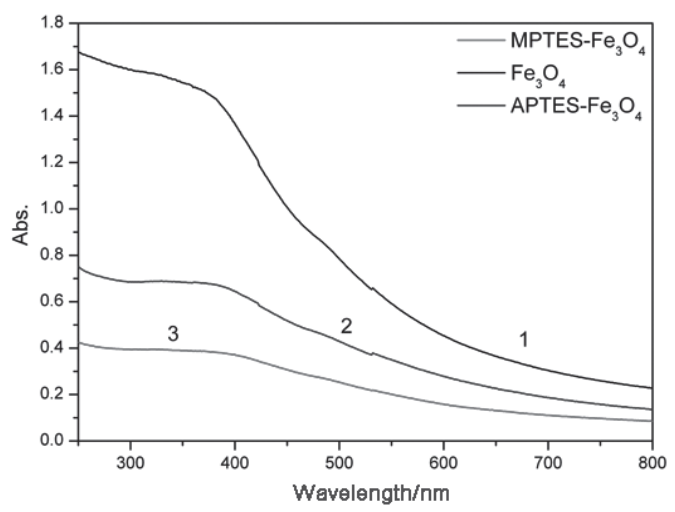

Fig. 7. UV-Vis spectra of bare (1), APTES-coated (2), and MPTES-coated (3) $\mathrm{Fe}_{3} \mathrm{O}_{4} \mathrm{NPs}$.
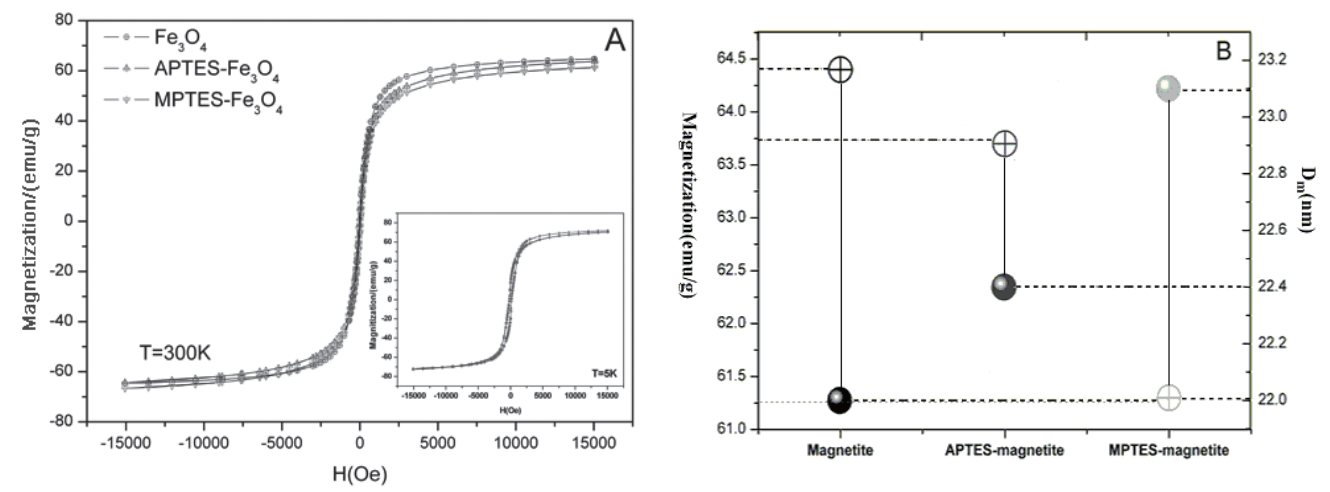

Fig. 8. Hysteresis loops of $\mathrm{Fe}_{3} \mathrm{O}_{4} \mathrm{NPs}$ and surface-functionalized $\mathrm{Fe}_{3} \mathrm{O}_{4} \mathrm{NPs}$ measured at $T=300$ $\mathrm{K}$ and $5 \mathrm{~K}(\mathrm{~A}), M_{\mathrm{S}}(\mathrm{emu} / \mathrm{g})(T=300 \mathrm{~K})$ and $D_{\mathrm{m}}(\mathrm{nm})(\mathrm{B})$.

decrease $M_{\mathrm{s}}$. The above interpretation in terms of surface anisotropy, as a result of the interaction with the silane-coated surface layers, suggests that surface anisotropy also affects the moment of the inner $\mathrm{Fe}_{3} \mathrm{O}_{4} \mathrm{NPs}$ via some exchange interactions at the surface. Thus, the $M_{\mathrm{s}}$ of the $\mathrm{Fe}_{3} \mathrm{O}_{4}$ NPs decreased with the immobilization of the APTES or MPTES on the surface of these NPs. Moreover, the decrease in magnetic property was very close to previously reported results. ${ }^{(17)}$ Therefore, the negligible loss in magnetic property enables further biochemical manipulation and assembly on surface tailored NPs towards biosensor applications.

In the absence of hysteresis, the average size of magnetic particles can be estimated from the initial susceptibility, $\chi_{i}=(\mathrm{d} M / \mathrm{d} H)_{H \rightarrow 0}$ coming mainly from the largest particles. The upper limit for the magnetic size, $D_{\mathrm{m}}$, may be estimated using the following eq. (3):(18) 


$$
D_{\mathrm{m}}=\frac{18 \mathrm{k}_{\mathrm{B}} T}{\pi} \frac{\chi_{i}}{\rho M_{\mathrm{s}}^{2}}
$$

where $\rho$ is the density of $\mathrm{Fe}_{3} \mathrm{O}_{4}\left(5.18 \mathrm{~g} / \mathrm{cm}^{3}\right)$ and $\mathrm{k}_{\mathrm{B}}$ is the Boltzmann constant. $\chi_{i}$ was determined approximately. By using saturation magnetization $M_{\mathrm{s}}$ obtained from the magnetization curve, $D_{\mathrm{m}}$ was estimated. Namely, the size diameter of the bare $\mathrm{Fe}_{3} \mathrm{O}_{4} \mathrm{NPs}$ was $22 \mathrm{~nm}$, that of the APTES-coated $\mathrm{Fe}_{3} \mathrm{O}_{4}$ NPs was $22.4 \mathrm{~nm}$, and that of the MPTEScoated $\mathrm{Fe}_{3} \mathrm{O}_{4} \mathrm{NPs}$ was $23.1 \mathrm{~nm}$ (shown in Fig. 8(B)), indicating good correlation between the particle sizes estimated from magnetization and TEM images.

\section{Conclusions}

In summary, we have reported an effective means of synthesizing silane-modified $\mathrm{Fe}_{3} \mathrm{O}_{4}$ NPs with amino-coated and thiolated magnetite NPs via surface APTES and MPTES functionalizations. The particles are completely characterized using a nanosizer, TEM, EDS, XRD analysis, and XPS, particularly UV spectrophotometry for optical property changes and SQUID for magnetic property changes. APTES was more prone to be fixed on the bare $\mathrm{Fe}_{3} \mathrm{O}_{4}$ surface, but the hypochromic effect of MPTES is stronger in comparison. The ultrafine silane-modified $\mathrm{Fe}_{3} \mathrm{O}_{4}$ nanospheres showed good dispersion and high saturation magnetization. These results reveal that the size of functionalized particles slightly increased; overlaying the $\mathrm{Fe}_{3} \mathrm{O}_{4} \mathrm{NP}$ surface with a shell of APTES or MPTES organic molecules results in negligible decrease in magnetic behavior. Moreover, the decrease in magnetic behavior was very close to that previously reported. The paramagnetic silane-modified $\mathrm{Fe}_{3} \mathrm{O}_{4}$ NPs are expected to be used for biomedical purposes.

\section{Acknowledgements}

The authors gratefully acknowledge the financial support from the Chinese 863 High-Tech Project (2006AA03Z357), the National Natural Science Foundation of China (60871007, 20505020), and the Scientific Research Fund of Hunan Provincial Education Department (08A013).

\section{References}

1 M. Amauraa, R. L. Camiloa, L. C. Sampaiob, M. A. Macedoc, M. Nakamurad and H. E. Toma: J. Magn. Magn. Mater. 279 (2004) 210.

2 J. Frenkel and J. Dorfman: Nature 126 (1930) 274.

3 M. Vázquez, C. Luna, M. P. Morales, R. Sanz, C. J. Serna and C. Mijangos: Phys. Rev. B: Condens. Matter 354 (2004) 71.

4 P. Tartaj, T. Gonzalez-Carreno and C. J. Serna: Adv. Mater. 13 (2001) 1620.

5 X. Battle and A. Labarta: J. Phys. D: Appl. Phys. 35 (2002) R15.

6 J. Wang, J. J. Sun, Q. Sun and Q. W. Chen: Mater. Res. Bull. 38 (2003) 1113.

7 D. K. Kim, Y. Zhang, J. Kehr, T. Klason, B. Bjelke and M. Muhammed: J. Magn. Magn. Mater. 225 (2001) 256. 
8 W. Wu, Q. G. He, H. Chen, J. X. Tang and L. B. Nie: Nanotechnology 18 (2007) 561.

9 D. K. Kim, M. Maria, F. H. Wang, K. Jan, B. Börje, Y. Zhang, T. Thomas and M. Mamoun: Chem. Mater. 15 (2003) 4343.

10 X. C. Shen, X. Z. Fang, Y. H. Zhou and H. Liang: Chem. Lett. 33 (2004) 1468.

11 J. F. Che, Y. Z. Xie, X. J. Yang, L. D. Lua and X. Wang: J. Nanjing University Science Technology 29 (2005) 330.

12 J. Wang, W. Wu and D. Feng: The Introduction to Electronic Spectroscopy (XPS/XAES/UPS) (Nation Defense Industry Press, Chinese, 1992) p. 519.

13 A. Schlegel, S. F. Alvarado and P. Wachter: J. Phys. C: Solid State Phys. 12 (1979) 1157.

14 Z. Simsa and K. Suk: Czech. J. Phys. 29 (1979) 593.

15 K. R. Brown, D. G. Walter and M. J. Natan: Chem. Mater. 12 (2000) 306.

16 A. M. Pyle, J. P. Rehmann, R. Meshoyrer, C. V. Kumar, N. J. Turro and J. K. Barton: J. Am. Chem. Soc. 111 (1989) 3051.

17 O'Handley and C. Robert: Modern Magnetic Materials: Principles and Applications (Wiley, New York, 1999) p. 768.

18 E. E. Carpenter: J. Magn. Magn. Mater. 225 (2001) 17. 